\title{
On the Data-Driven Road from Neurology to Neuronomy
}

\author{
Giorgio A. Ascoli ${ }^{1}$
}

Published online: 21 May 2016

(C) Springer Science+Business Media New York 2016

At the inaugural editorial board meeting of this journal some 15 years ago, one of the topics of discussion was how to measure overall success for the nascent field of neuroinformatics. The critical context for this question was the sprawling growth of the "NeuroX" vocabulary: not just neuroanatomy, neurophysiology, neurochemistry, neurocomputing, and neuroimaging, but also neuroethics, neuroevolution, neuroeconomics, neuropolicy, and neuroaesthetics. Among the most controversial ideas that were floated, it was predicted that, if fully successful, neuroinformatics would disappear as a subfield of neuroscience because all of neuroscience would become neuroinformatics. The rationale for such a seemingly preposterous proposals was rooted in one of the defining goals of neuroinformatics, namely to provide an information infrastructure for neuroscience. ${ }^{1}$ Thus, the argument went, when neuroscience becomes a sufficiently mature discipline to fully integrate experimental design and analysis, theory, and computational simulations, neuroinformatics will simply be the underlying framework for data flow among all approaches.

Much progress has been made since those early days, and several successful databases, digital resources, electronic tools, and virtual labs have been described in the pages of our journal and elsewhere. An encouraging proportion of this material is already being incorporated in

\footnotetext{
${ }^{1}$ Ascoli, G. A., De Schutter, E., \& Kennedy, D. N. (2003). An information science infrastructure for neuroscience. Neuroinformatics, 1(1), 1-2.

Giorgio A. Ascoli

ascoli@gmu.edu

1 Krasnow Institute for Advanced Study, George Mason University, 4400 University Dr, Fairfax, VA 22030, USA
}

foundational academic courses ${ }^{2}$ as well as specialized schools. Nevertheless, it is safe to state that much work still remains ahead. When the Society for Neuroscience "retired" its neuroinformatics committee after 7 years of operations in 2010, much of the needed planning role was overtaken by the International Neuroinformatics Coordinating Facility (INCF). Similarly, not too long after the National Institutes of Health ended its original "Human Brain Project", 3 neuroinformatics was featured prominently in the stated requirements of major community efforts such as the US BRAIN initiative, the EU Human Brain Project, and other international enterprises. ${ }^{4} \mathrm{~A}$ healthy sign of growing opportunities in this field is the emergence of alternative journals such as Frontiers in Neuroinformatics ${ }^{5}$ and more recently Brain Informatics. ${ }^{6}$ A similar multiplicative phenomenon is occurring with new international conferences being organized besides the INCF congress ${ }^{7}$ and the Brain Informatics and Health meeting. ${ }^{8}$

Social barriers are proving to remain harder to tear down than technical obstacles. Despite the continuously increasing shift towards acceptance and encouragement of neuroscience

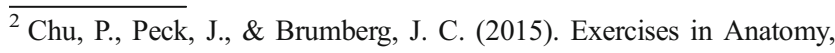
Connectivity, and Morphology using Neuromorpho.org and the Allen Brain Atlas. Journal of Undergraduate Neuroscience Education, 13(2), A95-A100.

${ }^{3}$ De Schutter, E., Ascoli, G. A., \& Kennedy, D. N. (2006). On the future of the human brain project. Neuroinformatics, 4(2), 129-130.

${ }^{4}$ Kandel, E. R., Markram, H., Matthews, P. M., Yuste, R., \& Koch, C. (2013). Neuroscience thinks big (and collaboratively). Nature Reviews. Neuroscience, 14(9), 659-664.

5 journal.frontiersin.org/journal/neuroinformatics

${ }^{6}$ springer.com/computer/ai/journal/40708

${ }^{7}$ neuroinformatics2016.org

${ }^{8}$ wibih.unomaha.edu/bih
} 
data sharing, ${ }^{9}$ major biomedical publications are still in 2016 conservatively contrasting "productive" (though un-shared) data collection versus "parasitic" (though open) data re-use. ${ }^{10}$ At least, the harsh criticisms that such a "retro" position promptly attracted through effective (and informaticsdriven... ${ }^{11}$ ) platforms for free scientific exchange ${ }^{12}$ resulted in a belated editorial clarification, ${ }^{13}$ offering another uplifting indication that the community is nevertheless moving in the right direction. Ultimately, availability and standardization of quantitative data is quintessential for rigorous scientific development and "evidence-based medicine" alike. Thus, neuroinformatics may be considered crucial for transitioning the still largely qualitative and descriptive field of neurology into a future predictive discipline of "neuronomy", though the parallel contrast between astrology and astronomy is certainly not meant to be interpreted literally.
While carrying on in the neuroinformatics pursuit of neuroscience progress, we maintain the tradition of rotating our journal editorial board composition. Although it is difficult to part with colleagues who have served since that very first editorial board meeting, over the course of the past 6 months we expressed our grateful farewell (also on behalf of our authors) to Ad Aertsen, Carrolee Barlow, Jan Bjaalie, Jonathan Cohen, Mark Ellisman, Peter Fox, Christiane Linster, Cynthia Moss, Miguel Nicolelis, Shigeru Tanaka, and Naftali Tishby. At the same time, we are happy to welcome Gaute Einevoll, Klaus Hilgetag, Mary Kennedy, Jynny Kim, Jeffrey Krichmar, Angela Laird, Jean-Baptiste Poline, Jessica Turner, Mark van Rossum, Daniel Wojcik, and Yoko Yamaguchi. The work of excellent scientists is instrumental toward making the Journal as successful as it has become, and the new contributors represent the diverse and highly dynamic nature of our field.

\footnotetext{
${ }^{9}$ Ascoli, G. A. (2014). A community spring for neuroscience data sharing. Neuroinformatics, 12(4), 509-511.

${ }^{10}$ Longo, D. L., \& Drazen, J. M. (2016). Data Sharing. The New England Journal of Medicine, 374(3), 276-277.

${ }^{11} \mathrm{https}: / /$ hypothes.is/about/

$12 \mathrm{https}$ //via.hypothes.is/http:/www.nejm.org/doi/full/10.1056/ NEJMe1516564

${ }^{13}$ Drazen JM. (2016). Data sharing and the journal. The New England Journal of Medicine. doi:10.1056/NEJMe1601087; PMID: 26,808,582.
} 